\title{
Logros y retos de la atención integral de personas con discapacidad víctimas del conflicto armado: aprendizajes de un proyecto de cooperación
}

Achievements and challenges of comprehensive care of people with
disabilities as victims of the armed conflict: lessons learned from a
cooperation project

Realizações e desafios do atendimento integral de pessoas com deficiência, vítimas de conflito armado: lições aprendidas em um

projeto de cooperação

Eliana Ibeth Sepúlveda Zabala'

Aleida Marcela Valbuena Pachón ${ }^{2}$

Recibido: 9 de abril 2019 • Enviado para modificación: 27 de diciembre 2019 • Aceptado: 4 de junio 2020

\section{RESUMEN}

Este artículo recoge y reflexiona alrededor de los logros y desafíos más significativos de un proyecto de cooperación implementado entre 2014 y 2017 en 12 ciudades y municipios de Colombia, que tuvo por objeto promover la independencia y la participación de personas con discapacidad víctimas del conflicto armado. Estos aprendizajes pueden ser analizados y tenidos en cuenta por gobiernos, tomadores de decisiones, profesionales y otros implementadores de programas para mejorar las políticas, programas y proyectos que favorezcan las condiciones de esta población. Se destacan aspectos relevantes de la participación de

1 Fisioterapeuta. Magíster en Administración en Salud. Magíster en Discapacidad e Inclusión Social. Instituto Colombiano de Bienestar Familiar. Bogotá, Colombia. eisepulvedaz@unal.edu.co. (iD) https://orcid.org/0000-0002-2758-3870

2 Terapeuta ocupacional. Especialista en Planeación, Control y Gestión del Desarrollo Social. Instituto Nacional para Ciegos -INCI. Bogotá, Colombia. mvalbuenap@gmail.com. (iD) https://orcid.org/0000-0002-5989-5975 
terapeutas ocupacionales en el campo de la inclusión social de personas con discapacidad en escenarios de posacuerdo, derivados de la experiencia del proyecto.

\title{
PALABRAS CLAVE
}

personas con discapacidades, medicina física y rehabilitación, conflictos armados

\begin{abstract}
This article presents the most significant achievements and challenges of a cooperation project implemented between 2014 and 2017 in 12 cities and municipalities of Colombia. The objective of this project was to promote the independence and participation of people with disabilities who were victims of the armed conflict. The lessons learned through the project can be analyzed and considered by governments, decision makers, professionals and other program implementers, to improve policies, programs and projects that support the conditions of this population. The article highlights some relevant aspects of the intervention of occupational therapists in the field of social inclusion of people with disabilities in post-agreement scenarios, derived from the project's experience.
\end{abstract}

\section{KEY WORDS}

disabled persons, physical and rehabilitation medicine, armed conflicts

\section{RESUMO}

Este artigo reúne as mais significativas realizações e desafios de um projeto de cooperação implementado entre 2014 e 2017 em 12 cidades e municípios da Colômbia. Teve como objetivo promover a independência e a participação de pessoas com deficiência, vítimas do conflito armado. Como lições de aprendizagem, eles podem ser analisados e levados em conta pelo governo, por tomadores de decisões, por profissionais e outros implementadores de programas, para aprimorar políticas e projetos que favoreçam as condições dessa população. O artigo destaca alguns aspectos relevante da intervenção do terapeutas ocupacionais no campo da inclusão social de pessoas com deficiência em cenários pós-contrato, derivados da experiência do projeto.

\section{PALAVRAS-CHAVE}

pessoas com deficiência, medicina física e reabilitação, conflitos armados

\section{Introducción}

El conflicto armado colombiano ha tenido múltiples efectos en la sociedad, entre ellos, secuelas físicas, emocionales y psicológicas en las numerosas víctimas de más de cinco décadas de confrontación violenta. Desde la Unidad para la Atención y Reparación Integral a las Víctimas (UARIV), se consideran víctimas con discapacidad "aquellas personas que además de ha- 
ber experimentado hechos victimizantes $^{3}$, presentan diversidad funcional de tipo físico, sensorial, intelectual, mental o múltiple, que al interactuar en la sociedad encuentran barreras que los excluyen en el ejercicio de sus dere$\operatorname{chos}^{\prime \prime}$ (2016, p.1). De acuerdo con el Registro Único de Víctimas (UARIV, 2020), para abril de 2020, alrededor de 360.000 personas estaban registradas como víctimas con discapacidad $(4,05 \%$ del total), cifra que no representa el total de esta población que se calcula ha sido afectada por el conflicto armado (Auto 173/14, 2014; Correa y Castro, 2016). De otra parte, existe evidencia de que en Colombia "la discapacidad se origina y exacerba por fenómenos como el conflicto armado" (UARIV, 2017, p.11).

El momento de posacuerdo que vive el país demanda que las víctimas, entre ellas las víctimas con discapacidad, sean reparadas y atendidas a través de acciones del Estado y la sociedad, y que se les garanticen sus derechos. Esto, además, se hace imperativo a la luz de la Convención sobre los Derechos de las Personas con Discapacidad (Organización de las Naciones Unidas - ONU, 2006) y algu- nos pronunciamientos de la Corte Constitucional (Sentencia T-025/04, 2004; Auto 006/09, 2009; Auto 173/14, 2014).

En este contexto, entre el 2014 y el 2017 se implementó en Colombia el proyecto TEAM, que convocó a once organizaciones del país ${ }^{4}$ para que, con recursos de cooperación de la Agencia Internacional para el Desarrollo de los Estados Unidos (USAID, por sus siglas en inglés), promovieran la independencia y plena participación de personas con discapacidad víctimas del conflicto armado, principalmente mujeres y niñas ${ }^{5}$.

Este artículo tiene como propósito reseñar y reflexionar alrededor de los principales logros y retos del proyecto ${ }^{6}$ en relación con la articulación de las políticas de discapacidad y de víctimas para la garantía de derechos, de acuerdo con las condiciones territoriales y poblacionales; la construcción de capacidad institucional a nivel local que puede generarse a partir de iniciativas de este tipo; el rol de las organizaciones de base comunitaria en procesos de inclusión de población con discapacidad víctima, y el fortalecimiento de la agencia de estas personas para la incidencia en la garan-

\footnotetext{
${ }^{3}$ De acuerdo con la Unidad para la Atención y Reparación Integral a las Víctimas "son hechos victimizantes: el abandono o despojo forzado de tierras, los actos terroristas, la amenaza, los combates, los enfrentamientos y hostigamientos, la violencia sexual, la desaparición forzada, el desplazamiento forzado, el homicidio, la masacre, los accidentes con minas antipersonal (MAP), las municiones sin explotar (MUSE) y los artefactos explosivos improvisados (AEI), el secuestro, la tortura, la vinculación de niños, niñas y adolescentes a actividades relacionadas con los grupos armados, así como los que determine la ley" $(2017$, p.5).

${ }^{4}$ Las organizaciones que participaron en el proyecto fueron: World Vision, Fundación CIREC, Instituto de Ortopedia Infantil Roosevelt, Fundación Saldarriaga Concha, Fundación Ser Social, Asociación Amigos con Calor Humano, Prodepaz, Servicios de Consultoría Integral, Fundación Arcángeles, Instituto Sur Alexander Von Humboldt y Fundación para la Estimulación en el Desarrollo y las Artes.

${ }^{5}$ Este propósito y énfasis en mujeres y niñas son propios de la concepción del proyecto por parte de USAID.

${ }^{6}$ Estos fueron recopilados y presentados en extenso en el documento Proyecto TEAM Colombia, una experiencia para aprender (Duarte y Moya, 2017), resultado de la sistematización del proceso al cierre del proyecto
} 
tía de sus derechos. De la misma forma, pretende destacar el rol profesional de Terapia Ocupacional en escenarios de posacuerdo, con el fin de aportar al fortalecimiento de esta labor. Lo anterior, a partir de la experiencia directa de las autoras en el diseño e implementación del proyecto desde la asesoría técnica y la coordinación regional del mismo.

\section{El proyecto}

El proyecto TEAM es una iniciativa financiada por la Agencia Internacional para el Desarrollo de los Estados Unidos de América (USAID) para incrementar la disponibilidad y el acceso a programas dirigidos a las personas con discapacidad en paises afectados por conflictos y que ha sido implementado en la República Democrática del Congo, República Democrática Popular Laos, Ucrania y Colombia (USAID, 2016).

El nombre del proyecto, TEAM, se genera a partir de las líneas de acción con las cuales fue concebido en inglés: training, economic empowerment, assistive technology y medical rehabilitation, y que constituyen los ejes a través de los cuales se desarrolla en los diferentes paises. En el contexto colombiano, dichas líneas se denominaron capacitación, empoderamiento económico, tecnología de asistencia y rehabilitación médica/física (Duarte y Moya, 2017), no obstante, el acrónimo TEAM se conserva en los diferentes paises en los que se implementa, independientemente del idioma local.
En Colombia, el proyecto TEAM fue gestado con el propósito de promover la independencia y la participación de las personas con discapacidad, de acuerdo con el enfoque de derechos que fue adoptado a través de la Ley 1346 (2009), con la cual se ratificó en el país la Convención sobre los Derechos de las Personas con Discapacidad (ONU, 2006), y con base en lo establecido en la artículo 13 de la Carta Constitucional (1991), en relación con la protección que el Estado debe proveer a esta población, como una forma de responder a la necesidad de garantizar sus derechos humanos, especialmente a aquellas víctimas del conflicto armado.

En este sentido, el proyecto buscó generar acciones que impactaran las diferentes esferas de la vida de las personas participantes y sus familias, como una apuesta de atención integral que involucró que los socios principales del proyecto $^{7}$ apropiaran y diseñaran las cuatro líneas de acción, así: a) formación en derechos, dirigida a la población con discapacidad para incrementar su capacidad de agencia, y a funcionarios públicos, con el fin de sensibilizarlos sobre los derechos de las personas con discapacidad; b) empoderamiento económico, para la generación de recursos como fuente de sustento de las personas con discapacidad y sus familias (apoyo y acompañamiento a emprendimientos productivos nuevos y existentes, y capacitación en empleabilidad); c) provisión de dispositivos de asistencia para aumentar la independencia de las personas con discapacidad y facilitar su participación social, y d) rehabilitación

\footnotetext{
${ }^{3}$ World Vision se desempeñó como administrador e implementador del proyecto, la Fundación CIREC, el Instituto de Ortopedia Infantil Roosevelt y la Fundación Saldarriaga Concha, como implementadores de las líneas de acción.
} 
física, con el fin de prestar atención con enfoque de rehabilitación integral, a través de brigadas móviles y servicios de telemedicina articulados con los prestadores de salud a nivel local. Este último componente incluyó acciones de formación al personal de salud, con miras a generar capacidades en las instituciones locales alrededor de temas técnicos en rehabilitación, administración de servicios de salud y calidad en la prestación de servicios.

El proyecto priorizó la atención en lugares del país que han sido afectados especialmente por el conflicto, en donde se concentra una gran cantidad de víctimas. Así, se implementó en cinco ciudades capitales: Barranquilla, MedeIlín, Bogotá, Cali y Pasto, que están entre las principales receptoras de personas desplazadas por cuenta del conflicto
(Centro Nacional de Memoria Histórica, 2015; Bohada, 2010), y siete municipios ubicados en cinco departamentos: Carmen de Bolívar y Ovejas, en Bolívar; San Carlos y San Francisco, en Antioquia; Soacha, en Cundinamarca; El Tambo, en Cauca, y Tumaco, en el departamento de Nariño. A excepción de Soacha, en estos municipios los porcentajes de víctimas, respecto al total de su población, superan el 65\% (Duarte y Moya, 2017).

En cuanto a las características de las personas con discapacidad participantes en el proyecto, de acuerdo con los datos reportados por Duarte y Moya (2017), la mayor parte de ellas fueron mujeres, menores de 18 años, residentes en zonas urbanas y pertenecientes al régimen subsidiado de salud, lo que obedece, en términos generales, al enfoque del proyecto (ver tabla 1 y figura 1 ).

Tabla 1. Tipo de discapacidad en participantes del proyecto TEAM Colombia.

\begin{tabular}{|l|c|}
\hline \multicolumn{1}{|c|}{ Tipo de discapacidad } & Porcentaje de participantes \\
\hline Física & $59 \%$ \\
\hline Sensorial & $9 \%$ \\
\hline Cognitiva & $12 \%$ \\
\hline Psicosocial & $7 \%$ \\
\hline Múltiple & $13 \%$ \\
\hline
\end{tabular}

Fuente: elaboración propia a partir de los datos reportados por Duarte y Moya (2017). 
Figura 1. Caracterización de participantes con discapacidad en el proyecto TEAM Colombia.

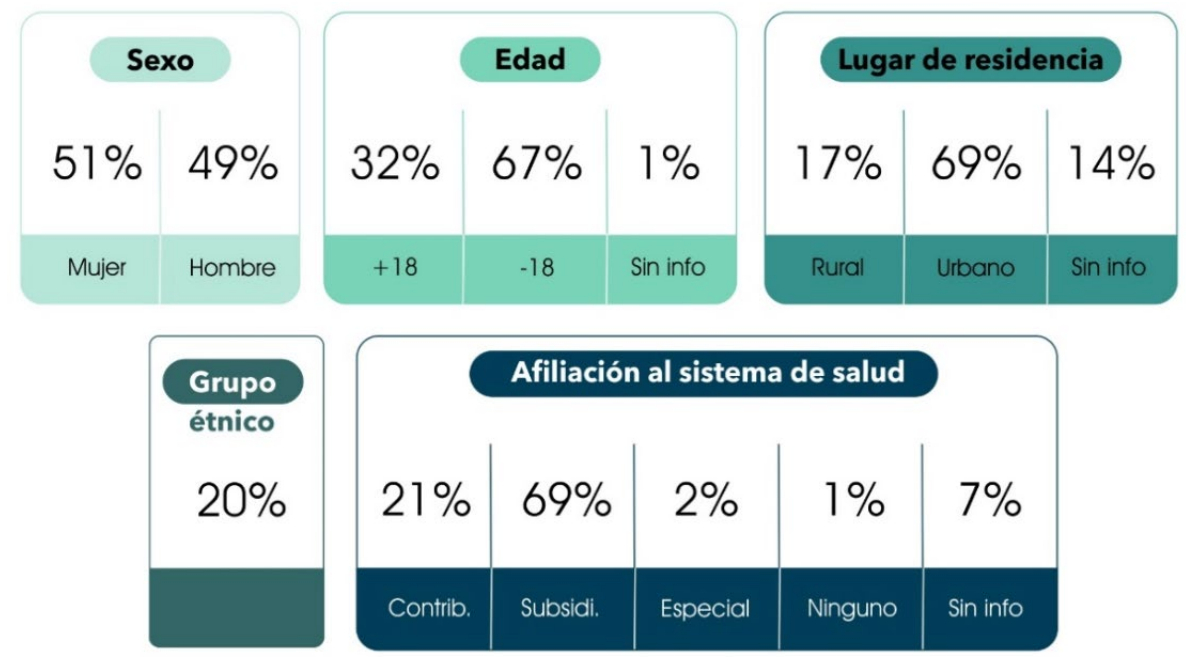

Fuente: elaboración propia a partir de los datos reportados por Duarte y Moya (2017).

\section{Logros y desafíos del proyecto TEAM}

\section{Principales logros alcanzados}

De acuerdo con los datos reportados por Duarte y Moya (2017), durante la implementación se logró involucrar, a través de los diferentes componentes, a 3.670 personas con discapacidad, con un promedio cercano a las 300 en cada ciudad o municipio. De ellas, solo el $35,6 \%$ estaban registradas en el Registro Único de Víctimas, las demás no se habían registrado o no se reconocían como tales, lo que da cuenta de limitaciones en los mecanismos de registro y en los reportes oficiales, pero también de necesidades de la personas con discapacidad, no necesariamente víctimas del conflicto, quienes históricamente han enfrentado barreras o no han sido atendidas por el sistema de salud y los programas sociales. De la misma forma, participaron más de 5.000 personas sin discapacidad, incluyendo familiares, personas cuidadoras, agentes comunitarios y otros miembros de la comunidad, así como profesionales de la salud (aproximadamente 1.200). El proyecto también impactó de forma indirecta alrededor de 15.300 personas que, aunque no estuvieron directamente involucradas, percibieron sus efectos.

Cabe destacar dos acciones claves realizadas en el proyecto para incrementar el acceso a servicios de salud y rehabilitación, el cual, como es bien conocido, tiene barreras importantes relacionadas con los trámites administrativos y la disponibilidad de servicios en zonas rurales o rurales dispersas de difícil acceso, pero también en áreas urbanas (Rodríguez et al., 2015; Vélez et al., 2016). Estas fueron: 
Orientación para la gestión de autorizaciones de órdenes médicas ante las Entidades Promotoras de Salud (EPS), a través del promotor en rutas de atención en salud. Se realizó orientación y asesoría individual a cada persona valorada en el componente de rehabilitación, en la ruta a seguir dentro del proyecto y a través del sistema de salud, para cumplir con su plan de tratamiento. En este proceso fue fundamental el apoyo de las y los profesionales en campo (en Psicología, Trabajo Social, Terapia Ocupacional), quienes hacían el seguimiento y el acompañamiento a las personas con discapacidad inscritas en su zona, identificando a aquellas que presentaban dificultades en el acceso a los servicios y refiriéndolas al promotor de rutas. Esta acción articulada incidió de manera importante en la adherencia a los servicios de rehabilitación prestados por el proyecto, logrando que un $75 \%$ de los participantes los finalizaran (Instituto de Ortopedia Infantil Roosevelt - IOIR, 2017).

De la misma forma, dicha orientación favoreció la prestación, por parte de las entidades promotoras de salud, de servicios prescritos desde el proyecto. A través de esta asesoría y orientación personalizada se logró que estas entidades atendieran el $25,8 \%$ de las prescripciones no cubiertas por el proyecto TEAM (IOIR, 2017). Aunque esta cifra es baja, resulta significativa, si se tiene en cuenta que se trataba de servicios que no habían sido identificados ni prescritos previamente por el sistema de salud; adicionalmente, constituyó una reactivación de la ruta de atención en salud para personas que no estaban siendo atendidas. No obstante, lo anterior revela limitaciones importantes en los servicios de rehabilitación que pres- ta el sistema de salud a las personas con discapacidad.

Por otro lado, tanto las y los profesionales en campo como el promotor de rutas realizaron un proceso de acompañamiento con la población atendida y sus familias para generar o fortalecer capacidades para la gestión autónoma de procedimientos y la exigibilidad de derechos, entre ellos, a la atención en salud. De manera particular, el abordaje en Terapia Ocupacional desplegó acciones para fomentar la independencia, aprovechar potencialidades y adquirir capacidades que permitieran a las personas con discapacidad participantes hacer las actividades por sí mismas, otorgándoles un sentido de competencia y capacidad que favoreció su inclusión.

Como acción complementaria, los equipos profesionales en campo y las organizaciones locales que participaron en la implementación del proyecto identificaron las rutas de acceso a servicios gubernamentales (justicia, atención en casos de violencia intrafamiliar o de género y maltrato infantil, ofertas recreo-deportivas) y a espacios de inclusión ofrecidos por otras instituciones locales en cada cuidad y municipio, y generaron acciones articuladas con varias de estas entidades, información que fue socializada con la población. Esto permitió a las personas participantes ampliar su conocimiento sobre los servicios a los que las personas con discapacidad y sus familias tienen derecho, y facilitó su reconocimiento e inclusión en espacios propios del territorio, diferentes a los ofertados por el proyecto TEAM.

Implementación de telemedicina como estrategia para mejorar el acceso a servicios de rehabilitación. A 
través del proyecto se implementó el servicio de telemedicina para hacer seguimiento y monitoreo a los procesos de rehabilitación prescritos desde TEAM y ejecutados por instituciones prestadoras de servicios de salud (IPS) locales, públicas y privadas. En este marco de trabajo, el Instituto de Ortopedia Infantil Roosevelt creó un modelo de intervención en telemedicina, incluyendo el diseño de un aplicativo y la estandarización de actividades para el montaje operacional de estaciones de telemedicina a nivel local. Dicha operación comprendió la capacitación a los equipos de las instituciones prestadoras de servicios de salud locales; el acompañamiento a estas instituciones para gestionar la habilitación de los servicios ante las entidades reguladoras en cada territorio; la verificación de los requerimientos humanos, técnicos y de comunicación para el uso del sistema de telemedicina, y la provisión y activación de equipos.

Bajo este modelo, se logró la habilitación por parte de las secretarías locales de salud de un centro de referencia en telemedicina en Bogotá y de nueve estaciones satélites en instituciones prestadoras de servicios de salud remisoras, a través de las cuales se atendió al total de participantes del componente de rehabilitación en algún momento de sus procesos.

Como estrategia para garantizar la sostenibilidad y la continuidad, posterior al cierre del proyecto, las instituciones recibieron acompañamiento para incluir el servicio dentro de su portafolio; se les asesoró en la definición de la oferta de acuerdo al perfil epidemiológico de la zona, en el diseño del manual tarifario, aspectos logísticos, la implementación de guías y protocolos de atención y la apertura de agendas de citas (IOIR, 2017).

\section{Desafíos más significativos}

Teniendo en cuenta la perspectiva integral del proyecto, la diversidad de la población y las características de los territorios priorizados, los retos más importantes que reveló su implementación pueden organizarse en cinco categorías, de acuerdo con las acciones en las que se presentaron mayores barreras, así: a) identificación de la población participante; b) articulación con actores locales; c) procesos de formación; d) empoderamiento económico, y e) procesos de atención en salud y rehabilitación.

Identificación de población con discapacidad víctima del conflicto. Como primera fuente para la ubicación de potenciales participantes con discapacidad, se acudió al Registro de Localización y Caracterización de la Personas con Discapacidad y al Registro Único de Víctimas. No obstante, debido al subregistro, la dispersión y la desactualización de la información en el primero y las dificultades para acceder al segundo, esta estrategia resultó insuficiente (Duarte y Moya, 2017).

De igual manera, se intentaba llegar a la población con discapacidad que no hubiese participado en otros proyectos en la zona, sin embargo, no existían registros ni datos actualizados al respecto, especialmente en relación con la entrega de dispositivos de asistencia o proyectos de emprendimiento. En consecuencia, fue necesario recurrir a la búsqueda activa y a organizaciones de base comunitaria, como colectivos de personas con discapacidad e instituciones que ofertan servicios a la población 
con discapacidad y a víctimas, las cuales participaron en este proyecto como implementadores en las regiones.

En relación con el énfasis en la atención a mujeres y niñas, ello también se convirtió en un desafío, pues los resultados parciales durante la ejecución del proyecto mostraban un equilibrio entre mujeres y hombres. Al cierre de proyecto se logró que las participantes con discapacidad fuesen el $51 \%$, lo cual coincide con la distribución que ofrece del Registro de Localización y Caracterización (Ministerio de Salud, 2018). Sin embargo, se identificó que el $67 \%$ de las personas sin discapacidad participantes fueron mujeres, tanto en el rol de cuidadoras como profesionales de rehabilitación (Duarte y Moya, 2017). Este hecho confirma que las labores de cuidado, tanto formal (dentro de las cuales se puede incluir la rehabilitación) como informal, continúan teniendo una carga de género importante. Este rol adicional en la vida de las mujeres generalmente no es reconocido ni valorado y demanda procesos de acompañamiento para reducir los riesgos del cuidador quemado (Duarte y Hernández, 2019).

Articulación con actores locales, con el fin de fortalecer capacidades en los territorios y garantizar la sostenibilidad de las acciones. Al respecto, como se ha mencionado, el proyecto involucró para su operación a organizaciones de personas con discapacidad y a organizaciones de emprendimiento económico locales. Esta decisión generó confianza y facilitó el involucramiento de la población, pues estas organizaciones desarrollaron modelos de atención fundamentados en su propia experiencia, en las características de la personas y grupos, y en las dinámicas del territorio. Como resultado, se lograron acciones pertinentes, así como una mayor permanencia y participación efectiva en los procesos. Por otra parte, el proyecto aseguró la articulación con los gobiernos locales, las secretarías de salud, los referentes de discapacidad, la Unidad de Víctimas y las entidades promotoras de salud, entre otras, lo que facilitó el desarrollo de las acciones en las ciudades y municipios, y es clave también para el fortalecimiento de capacidades, el aprovechamiento de recursos y la continuidad de las acciones.

Estas acciones de articulación, en particular el trabajo con hospitales de la red pública, se vio afectado por la insuficiencia de recursos humanos y de equipamiento para los servicios de rehabilitación; ello implicó desafíos significativos para asegurar la atención de las personas participantes, principalmente en los municipios pequeños. Por ejemplo, no todos los hospitales públicos contactados contaban con servicios de rehabilitación, y aquellos que los tenían solamente disponían de talento humano en Fisioterapia para la atención. Ello puso en evidencia la concentración de profesionales de Terapia Ocupacional y Fonoaudiología en ciudades capitales. Para superar este hecho, el proyecto tuvo que desplazar profesionales en Terapia Ocupacional y Fonoaudiología desde Bogotá para trabajar en equipos itinerantes en los territorios.

Procesos de formación. En este componente se identificaron tres retos importantes, el primero, lograr que la comunidad acogiera los procesos de formación en derechos, discapacidad e inclusión para gestar cambios a partir del trabajo conjunto de diferentes actores en estos espacios. 
La diversidad de la población participante en este componente demandó el uso de metodologías y estrategias pedagógicas y didácticas innovadoras y diversas en cada territorio, a partir de las realidades locales. Fue evidente que el uso reiterado de talleres en diferentes proyectos dirigidos a poblaciones vulnerables ha agotado su utilidad y a las personas. En este sentido, las organizaciones locales implementaron visitas individuales a las familias para asesoría personalizada e incluyeron temas adicionales a los definidos en la estructura temática inicial, por ejemplo, aquellos relacionados con cultura de paz y construcción de planes de vida, con el fin de responder a las necesidades de las comunidades en cada territorio y a sus propios saberes (Duarte y Moya, 2017).

A su vez, con el fin de tender puentes entre la comunidad y la institucionalidad en los territorios para la construcción de planes de acción conjuntos, orientados a la garantía de derechos, se implementó la metodología Ciudadanía, Voz y Acción, a través de la cual las comunidades realizan acciones de defensoría a nivel local para transformar el diálogo con los gobiernos (World Vision International, 2012).

En este ámbito, las y los terapeutas ocupacionales en campo acudieron a la estrategia de rehabilitación basada en comunidad ( $R B C$ ) para acompañar a la comunidad en el análisis de los diferentes escenarios de participación de la población con discapacidad, favoreciendo la identificación de la oferta local y el acceso a servicios, y facilitando procesos de inclusión social y la participación en actividades significativas.

Un segundo reto fue la formación para mejorar la calidad de vida de las personas cuidadoras, reconociendo la integralidad de la atención a las personas con discapacidad y sus familias y el hecho de que esta es una necesidad subatendida en el país. Las y los profesionales en Terapia Ocupacional realizaron en todos los lugares de implementación encuentros con personas cuidadoras, en donde se abordaron temas de autocuidado, promoción de la salud física y mental, y promoción de la independencia y la autonomía de las personas con discapacidad, ello con el fin de fomentar capacidades, reducir la sobrecarga por las labores de cuidado y realizar modificaciones ambientales básicas que facilitaran su rol. La participación de terapeutas ocupacionales en este campo permitió sensibilizar a las y los cuidadores respecto a la importancia de buscar un equilibrio en su desempeño e implementar herramientas socio-emocionales (por ejemplo, espacios de recreación y esparcimiento personal, redistribución de funciones con otros miembros de la familia, identificación de redes de apoyo) que incidieran en su bienestar y en el de las personas con discapacidad.

Otro reto en formación fue el mejoramiento de las capacidades técnicas en rehabilitación en los territorios. Para facilitar el acceso, fue preciso desplegar tres modalidades de capacitación: presencial, virtual y pasantía hospitalaria, orientadas a mejorar la calidad de la atención en las ciudades y municipios. Esta estrategia permitió responder a las necesidades locales de formación continua y actualización en temas como salud mental, rehabilitación integral con enfoque biopsicosocial, lineamientos de rehabilitación basada en comunidad y provisión de sillas de ruedas bajo lineamientos de la Organización Mundial de la Salud, entre otros. 
A su vez, el Instituto de Ortopedia Infantil Roosevelt y World Vision ${ }^{8}$, junto con las y los profesionales en Terapia Ocupacional, articularon acciones con instituciones locales para ampliar sus capacidades en la provisión de dispositivos de asistencia, en términos de formación del recurso humano para la identificación, selección, entrenamiento y entrega de los mismos, de acuerdo con la evaluación de necesidades y el entorno de la persona con discapacidad, con miras a facilitar su independencia en actividades de la vida diaria y promover su inclusión social.

Empoderamiento económico. En relación con este componente, se destacan dos desafíos que enfrentó el proyecto. El primero de ellos, la existencia de una brecha importante entre los perfiles de las personas participantes que querían optar por el empleo como fuente de ingresos y los requisitos exigidos en las ofertas disponibles; la mayoría de ellas no habían finalizado la educación básica, que era el requisito mínimo de formación exigido, y aquellas con escolaridad básica o técnica no contaban con la experiencia laboral solicitada. En este sentido, en la ciudad de Pasto el proyecto gestionó una alianza con la caja de compensación familiar del departamento para promover procesos de formación para el trabajo de las personas con discapacidad participantes.

Un segundo desafío en este componente fue la necesidad de acciones de entrenamiento y adaptación de puestos de trabajo. A través del proyecto, 18 personas con discapacidad lograron una vinculación laboral (Duarte y Moya, 2017), sin embargo, para lograr el éxito y la permanencia en el empleo y estimular la inclusión laboral, se evidenció la necesidad de que dichos procesos incluyan acciones de capacitación y acompañamiento a empleadores y empleados para la adaptación de los puestos de trabajo antes de la vinculación, una vez se logra el enganche, durante el entrenamiento e, incluso, cuando se está considerando la desvinculación. En el sur del país, el proyecto identificó el interés de varias empresas en vincular a personas con discapacidad, por lo cual se gestionó una alianza con el programa de Terapia Ocupacional de la Universidad Mariana, en la ciudad de Pasto, para asesorar a las y los empleadores a partir de la identificación de intereses y capacidades de las personas con discapacidad en relación con el análisis de los requerimientos y exigencias de los puestos de trabajo. Con ello se buscó promover procesos de inclusión laboral efectivos, que respondieran no solo a las necesidades de las empresas sino a las expectativas de las personas con discapacidad.

Procesos de atención en salud y rehabilitación. Como se ha mencionado, este componente resultó ser uno de los más críticos, pues se vio afectado directamente por las problemáticas propias del sistema de salud del país. Dos de los desafíos más importantes fueron la provisión de servicios de rehabilitación en sí misma, pues en principio se contempló la vinculación de instituciones prestadoras de servicios de salud (IPS) en las regiones para el cumplimiento de los planes de tratamiento prescritos desde el proyecto; sin embargo, esto no se logró de manera oportuna debido a la ausencia de servicios de rehabilitación habilitados en los municipios. Así, los

\footnotetext{
${ }^{8} \mathrm{~A}$ través de un convenio con United Cerebral Palsy (UCP).
} 
convenios con los prestadores locales se materializaron solo hasta mediados del segundo año de implementación.

Por esta razón, en segunda instancia se optó por involucrar, capacitar y acompañar de manera directa a equipos profesionales en rehabilitación para atender a la población participante en cada una de los lugares priorizados; aun así, la insuficiencia de profesionales en los territorios hizo que esta modalidad no pudiese ser implementada.

Como último recurso, y sumado a las dificultades para el desplazamiento de las personas participantes desde las zonas rurales dispersas hacia los centros urbanos, se decidió llevar equipos itinerantes desde Bogotá hasta las regiones para realizar los procesos terapéuticos. Estos equipos lograron prestar los servicios de rehabilitación en instituciones ubicadas en los centros urbanos y a través de atención domiciliaria que incluyó sesiones de tratamiento, planes caseros y acciones de prevención y promoción.

En el último año de implementación, el proyecto logró cerrar del $75 \%$ de los procesos de rehabilitación a través de convenios con prestadores locales y la modalidad de equipos itinerantes, en ambos casos se usó telemedicina para el seguimiento, monitoreo y cierre. Los demás casos no lograron ser cerrados por razones como la atención directa por parte de la entidad promotora de salud (EPS), fallecimiento, traslado de ciudad de residencia, falta de interés de la persona o su familia y dificultades en el acceso geográfico que no pudo ser superada.
Atención en salud mental. A partir de la valoración integral en salud realizada en el proyecto, el 98\% de las personas con discapacidad participantes fueron remitidas a servicios de salud mental (Duarte y Moya, 2017). Debido a que el proyecto no incluía estas especialidades, se derivaron a las entidades promotoras de salud; sin embargo, fue evidente la necesidad de fortalecer la atención psicosocial a nivel local, pues en muchos casos esta demanda no fue satisfecha.

\section{Discusión}

Lo expuesto anteriormente permite evidenciar que aunque existen mandatos normativos que favorecen la protección de la población ${ }^{9}$, hay brechas en la atención diferencial y especializada para las personas con discapacidad, quienes están en riesgo de que su condición se incremente debido a la discriminación y a la exclusión en los servicios sociales y a las barreras para acceder a ellos, así como por la vulnerabilidad incrementada por la pérdida de la red social y familiar de apoyo como consecuencia del conflicto (Biel y Bolaños, 2018a).

En este contexto, la actual etapa de posacuerdo en la que se encuentra el país y la posible pérdida de vigencia de la Ley de Víctimas (Ley 1448, 2011) demandan el fortalecimiento del trabajo articulado entre el Sistema Nacional de Atención y Reparación Integral a Victimas y el Sistema Nacional de Discapacidad, con base en el enfoque de

\footnotetext{
${ }^{9}$ Entre ellos, el artículo 13 de la Constitución Política (1991), el Auto 006 de la Corte Constitucional (2009) y la Ley 1346 (2009).
} 
derechos. Adicionalmente, con el fin de garantizar los derechos de las personas con discapacidad víctimas del conflicto, es necesario que estos sistemas entren en diálogo con el Sistema Integral de Verdad, Justicia, Reparación y No Repetición, establecido en el Acuerdo Final de Paz (Gobierno Nacional y FARC-EP, 2016), teniendo en cuenta que además de las barreras existentes para el acceso a salud y a oportunidades laborales, esta población también enfrenta barreras para acceder a la representación legal y a la protección en el marco del Acuerdo (Biel y Bolaños, 2018b).

Así, los logros y desafíos de esta experiencia de fortalecimiento de la atención integral para la inclusión de personas con discapacidad víctimas del conflicto armado representa un aprendizaje significativo para el país y principalmente para los territorios, como una apuesta para las acciones a emprender.

De manera particular, la orientación y asesoría personalizada y permanente prestada a través del promotor en rutas de atención en salud constituye una estrategia que facilita el acceso a los servicios y el tránsito por el sistema de salud, y permite a las y los usuarios conocer $y$ exigir sus derechos.

Como estrategia complementaria para incrementar el acceso a servicios de salud, la experiencia del proyecto en el uso de telemedicina se convierte en una oportunidad para enfrentar las limitaciones en la oferta en zonas urbanas y rurales, las barreras de acceso geográfico y la escasez de profesionales y especialistas en algunos territorios. De la misma forma, esta modalidad de atención puede disminuir los costos y las dificultades asociadas al desplazamiento de usuarios a centros de atención en las ciudades.

De otro lado, teniendo en cuenta los desafíos relacionados con la identificación y localización de la población con discapacidad en el país, resulta valioso para la implementación de políticas, proyectos y programas la expedición de la Resolución 113 (2020), que normatiza la expedición del certificado de discapacidad y el registro de localización y caracterización. Su puesta en funcionamiento constituye un avance importante en la gestión de la información y un elemento adicional para dar cumplimiento a la Convención sobre los Derechos de las Personas con Discapacidad (ONU, 2006, artículo 31).

De la misma forma, el fortalecimiento de la atención psicosocial se configura como uno de los principales retos para el país, teniendo en cuenta que las víctimas del conflicto armado son "sustancialmente más propensas a sufrir trastornos de salud mental" (Cuartas et al., 2019, p.708). En este sentido, el proyecto evidenció las necesidades de atención de la población participante, lo que demanda respuestas urgentes por parte de los gobiernos locales para el fortalecimiento del Programa de Atención Psicosocial y Salud Integral a Víctimas del Conflicto Armado (PAPSIVI), con el fin de atender las afectaciones emocionales y psicosociales derivadas del conflicto armado. Es importante que estas respuestas incluyan la provisión de servicios de salud mental de calidad y atiendan a determinantes sociales como el acceso al trabajo formal, reduciendo las desigualdades en salud mental en Colombia (Cuartas et al., 2019).

Por último, uno de los factores determinantes en el acceso de las personas con 
discapacidad a la atención integral es la ausencia de servicios intersectoriales en zonas rurales y rurales dispersas, principalmente aquellos relacionados con salud, educación y trabajo. En relación con la atención en salud, la intervención de equipos multidisciplinarios itinerantes y la implementación de estrategias de telemedicina, como los implementados en el proyecto, pueden ser una respuesta para disminuir esta brecha.

\section{Conclusiones}

Los aprendizajes derivados de esta experiencia se convierten en posibilidades para avanzar en la materialización e implementación en los territorios de las políticas dirigidas a la población con discapacidad o víctima del conflicto, pues se orientan al fortalecimiento de las capacidades instaladas, pueden incidir positivamente en la institucionalidad local y consideran la sostenibilidad de las acciones emprendidas en los proyectos de cooperación.

De igual manera, estas iniciativas permiten develar necesidades en los territorios, a partir de experiencias y cifras concretas que, al ser socializadas con autoridades y tomadores de decisiones a nivel nacional y local, pueden generar cambios que se traduzcan en bienestar para las comunidades.

En la misma línea, retomando a Duarte y Moya (2017), se recomienda trabajar de la mano con profesionales y organizaciones locales, pues su conocimiento y experiencia en los territorios y con las comunidades permite disminuir las brechas culturales y fortalecer las capacidades locales para dar sostenibilidad a los procesos, una vez los proyectos terminan.
Para finalizar, como aprendizaje particular en relación con el rol de las y los profesionales de rehabilitación en el actual escenario de posacuerdo, especialmente terapeutas ocupacionales, se destacan tres aspectos: primero, la pertinencia de su participación en procesos de inclusión laboral, particularmente en la asesoría para la adaptación de puestos de trabajo; segundo, la importancia de su intervención, junto con otros profesionales de la salud y la rehabilitación, en el diseño y el fortalecimiento de programas encaminados al cuidado de las personas cuidadoras, quienes, a pesar de los avances en la normatividad y en la atención a la población con discapacidad en el país, continúan siendo invisibilizadas, y tercero, la creación de estrategias e incentivos desde varios sectores para favorecer el establecimiento de profesionales de salud y rehabilitación a lo largo y ancho del territorio nacional. 


\section{Referencias}

Agencia Internacional para el Desarrollo de los Estados Unidos [USAID] (2016). Training, economic empowerment, assistive technologies and medical rehabilitation (TEAM). https://www.usaid. gov/sites/default/files/documents/1861/ FS TEAM August 2016 0.pdf

Asamblea Nacional Constituyente (1991, 20 de julio). Constitución Política de la República de Colombia. Gaceta Constitucional No. 116. http://www.secretariasenado.gov.co/senado/basedoc/constitucion politica 1991.html

Biel, I. y Bolaños, T. (2018a). Persons with disabilities and the Colombian armed conflict. Disability \& Society, 33(3), 487491. https://doi.org/10.1080/09687599.2 $\underline{018.1423914}$

Biel, I. y Bolaños, T. (2018b). Are persons with disabilities included in the Colombian peace process? Disability \& Society, 33(4), 638-643. https://doi.org/10.1080/0 $\underline{9687599.2018 .1423940}$

Bohada, M.P. (2010). Desplazamiento forzado y condiciones de vida de las comunidades de destino: el caso de Pasto, Nariño. Revista de Economía Institucional, 12(23), 259-298. https://revistas.uexternado.edu.co/index.php/ecoins/article/ view/2507

Centro Nacional de Memoria Histórica (2015). Una nación desplazada: informe nacional del desplazamiento forzado en Colombia. CNMH y UARIV. http://www.centrodememoriahistorica. gov.co/descargas/informes-accesibles/ una-nacion-desplazada_accesible.pdf
Congreso de la República (2009, 31 de julio). Ley 1346 de 2009. Por medio de la cual se aprueba la Convención sobre los Derechos de las personas con Discapacidad. Diario Oficial No. 47.427. http:// www.alcaldiabogota.gov.co/sisjur/nor$\underline{\text { mas/Norma1.jsp?i }=37150}$

Congreso de la República (2011, 10 de junio). Ley 1448 de 2011. Por la cual se dictan medidas de atención, asistencia y reparación integral a las víctimas del conflicto armado interno y se dictan otras disposiciones. Diario Oficial No. 48.096. http://www.secretariasenado.gov. co/senado/basedoc/ley 1448 2011.html

Correa, L. y Castro M. (2016). Discapacidad e inclusión social en Colombia. Informe alternativo de la Fundación Saldarriaga Concha al Comité de Naciones Unidas sobre los derechos de las personas con discapacidad. Fundación Saldarriaga Concha. https://www.saldarriagaconcha. org/wp-content/uploads/2019/01/FSC Informe Alternativo Accesible Final.pdf

Corte Constitucional (2004, 22 de enero). Sentencia T-025/04 (Manuel José Cepeda Espinosa, M.P.). http://www.corteconstitucional.gov.co/relatoria/2004/t-025-04. htm

Corte Constitucional (2009, 26 de enero). Auto 006/09 (Manuel José Cepeda Espinosa, M.P.). http://www.corteconstitucional.gov.co/relatoria/autos/2009/a006-09. $\underline{\mathrm{htm}}$

Corte Constitucional (2014, 6 de junio). Auto 173/14 (Luis Ernesto Vargas Silva, M.P.). https://www.corteconstitucional.gov.co/ relatoria/autos/2014/A173-14.htm 
Cuartas, J., Liévano, L., Martínez, M. y Hessel, P. (2019). The invisible wounds of five decades of armed conflict: inequalities in mental health and their determinants in Colombia. International Journal of Public Health, 64, 703-711. https://doi. org/10.1007/s00038-019-01248-7

Duarte, C. y Moya, D. (2017). Proyecto TEAM Colombia: Una experiencia para aprender. World Vision Colombia. https://s3.amazonaws.com/publicaciones-worldvision/Proyecto + Team $+\mathrm{fi}-$ nal+LR.pdf

Duarte, C.H. y Hernández, A.M. (2019). Cuidado informal y discapacidad en Colombia: una perspectiva de género. Revista Latinoamericana en Discapacidad, Sociedad y Derechos Humanos, 3(2), 139150. http://redcdpd.net/revista/index.php/ revista/article/viewFile/166/94

Gobierno Nacional y FARC-EP (2016). Acuerdo final para la terminación del conflicto y la construcción de una paz estable y duradera. https://www.cancilleria.gov. co/sites/default/files/Fotos2016/12.11 1. 2016nuevoacuerdofinal.pdf

Instituto de Ortopedia Infantil Roosevelt [IOIR] (2017). Proyecto TEAM Colombia 2014 2017. Memorias epidemiológicas. IOIR.

Ministerio de Salud y Protección Social (2018). Sala situacional de las Personas con Discapacidad. https://www.minsalud.gov.co/sites/rid/Lists/BibliotecaDigital/RIDE/DE/PS/sala-situacional-discapacidad-junio-2018.pdf

Ministerio de Salud y Protección Social (2020, 31 de enero). Resolución 113 de 2020. Por la cual se dictan disposiciones en relación con la certificación de discapacidad y el Registro de Localización y Caracterización de Personas con Discapacidad. Diario Oficial No. 51.213. https://www.alcaldiabogota.gov.co/sisjur/ normas/Norma1.jsp?i=90389\&dt=S

Organización de las Naciones Unidas [ONU] (2006). Convención sobre los Derechos de las personas con Discapacidad. https://www.un.org/disabilities/documents/ convention/convoptprot-s.pdf

Rodríguez, J.M., Rodríguez, D.P. y Corrales, J.C. (2015). Barreras de acceso administrativo a los servicios de salud en población Colombiana, 2013. Ciência \& Saúde Colectiva, 20(6), 1947-1958. https://doi. org/10.1590/1413-81232015206.12122014

Unidad para la Atención y Reparación Integral a las Víctimas [UARIV] (2016). Víctimas en situación de discapacidad. En Participaz... La ruta de los derechos (capítulo 18). https://repositoryoim.org/ bitstream/handle/20.500.11788/975/ Capitulo\%2018.pdf? sequence=isAllowed $=y 21 \&$

Unidad para la Atención y Reparación Integral a las Víctimas [UARIV] (2017). Personas con discapacidad. http://www.unidadvictimas.gov.co/sites/default/files/documentosbiblioteca/discapacidad_0.pdf

Unidad para la Atención y Reparación Integral a las Víctimas [UARIV] (2020). Registro único de víctimas. https://www. unidadvictimas.gov.co/es/registro-unico-de-victimas-ruv/37394

Vélez, N.P., González, C.D., Velásquez, A.P. (2016). Revisión de las barreras de acceso a los servicios de salud de la población con discapacidad en Colombia entre los años 2005 a 2015. Revista CES Derecho, 7(2), 72-83. http://dx.doi. org/10.21615/cesder.7.2.5 
World Vision International (2012). Guía de campo de la Ciudadanía, Voz y Acción. Oficina Regional para América Latina y el Caribe. 